\title{
Polar observations of electron density distribution in the Earth's magnetosphere. 1. Statistical results
}

\author{
H. Laakso ${ }^{1}$, R. Pfaff ${ }^{2}$, and P. Janhunen ${ }^{3}$ \\ ${ }^{1}$ ESA Space Science Department, Noordwijk, Netherlands \\ ${ }^{2}$ NASA Goddard Space Flight Center, Code 696, Greenbelt, MD, USA \\ ${ }^{3}$ Finnish Meteorological Institute, Geophysics Research, Helsinki, Sweden
}

Received: 3 September 2001 - Revised: 3 July 2002 - Accepted: 10 July 2002

\begin{abstract}
Forty-five months of continuous spacecraft potential measurements from the Polar satellite are used to study the average electron density in the magnetosphere and its dependence on geomagnetic activity and season. These measurements offer a straightforward, passive method for monitoring the total electron density in the magnetosphere, with high time resolution and a density range that covers many orders of magnitude. Within its polar orbit with geocentric perigee and apogee of 1.8 and $9.0 R_{E}$, respectively, Polar encounters a number of key plasma regions of the magnetosphere, such as the polar cap, cusp, plasmapause, and auroral zone that are clearly identified in the statistical averages presented here. The polar cap density behaves quite systematically with season. At low distance $\left(\sim 2 R_{E}\right)$, the density is an order of magnitude higher in summer than in winter; at high distance $\left(>4 R_{E}\right.$ ), the variation is somewhat smaller. Along a magnetic field line the density declines between these two altitudes by a factor of 10-20 in winter and by a factor of 200-1000 in summer. A likely explanation for the large gradient in the summer is a high density of heavy ions that are gravitationally bound in the low-altitude polar cap. The geomagnetic effects are also significant in the polar cap, with the average density being an order of magnitude larger for high $K_{p}$; for an individual case, the polar cap density may increase even more dramatically. The plasma density in the cusp is controlled primarily by the solar wind variables, but nevertheless, they can be characterized to some extent in terms of the $K_{p}$ index. We also investigate the local time variation of the average density at the geosynchronous distance that appears to be in accordance with previous geostationary observations. The average density decreases with increasing $K_{p}$ at all MLT sectors, except at 14-17 MLT, where the average density remains constant. At all MLT sectors the range of the density varies by more than 3 orders of magnitude, since the geostationary orbit may cut through different plasma regions, such as the plasma sheet, trough, and plasmasphere.
\end{abstract}

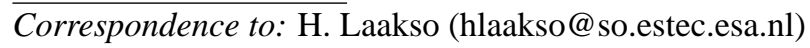

Key words. Magnetospheric physics (magnetospheric configuration and dynamics; plasmasphere; polar cap phenomena)

\section{Introduction}

The total electron number density is a key parameter needed for both characterizing and understanding the structure and dynamics of the Earth's magnetosphere. The spatial variation of the electron density and its temporal evolution during different geomagnetic conditions and seasons reveal numerous fundamental processes concerning the Earth's space environment and how it responds to energy and momentum transfer, both in response to the solar wind, as well as to internal sources, such as the ionosphere (e.g. Moore and Delcourt, 1995). Although the electron density in the magnetosphere is known in a general sense from case studies, large-scale, systematic studies of the electron density distribution in the magnetosphere have been performed only a few times in the past (e.g. Escoubet et al., 1997; Laakso et al., 1997; Johnson et al., 2001). Consequently, the models of the magnetospheric plasma density are much poorer than those for the magnetic field. The latter have been under development for many decades (e.g. Tsyganenko and Stern, 1996) and have benefited from the fact that almost every scientific satellite in the magnetosphere has carried a magnetometer. Plasma density measurements are more difficult to obtain in a systematic sense from a single instrument, since the plasma density and temperature might typically vary over several orders of magnitude.

There are a number of techniques for measuring the total plasma number density, but each technique has its own limitations. Spacecraft charging seriously limits the direct detection of low-energy charged particles and must be taken into account for a variety of instruments. Furthermore, photoelectrons and secondary electrons can cause a significant increase 
in the density around the spacecraft which degrades the quality of low-energy electron density measurements. Plasma wave cutoffs and resonances can provide excellent measurements of the plasma density if such waves are detectable, which is not always the case in tenuous plasmas. Also, sometimes, these waves do not represent the local plasma where the satellite is located. Electric field double probe instruments (EFI) provide measurements of the potential difference between a sensor and the satellite body, which depend on the total electron density, as we discuss below.

In addition to electric field measurements, electric field double probes typically monitor the potential difference $(\Delta V)$ between each sensor and the satellite (Mozer et al., 1978; Pedersen et al., 1978). In the case of the Polar satellite, the probe potential is kept artificially near the local plasma potential by means of a bias current. The measured potential difference, therefore, is approximately the spacecraft potential (with respect to the plasma potential) which is, on the other hand, proportional to the electron density and only weakly dependent on the electron temperature (Laakso and Pedersen, 1998). Therefore, $\Delta V$ measurements offer a straightforward, passive method for monitoring the variation of the total electron density in the magnetosphere with a high time resolution (e.g. less than $1 \mathrm{msec}$ ) and a range that covers many orders of magnitude. The accuracy of this technique remains valid even for densities well below $1 \mathrm{~cm}^{-3}$, as shown by numerous in situ measurements in the magnetosphere (e.g. Pedersen, 1995, and references therein). This technique has been applied in several studies of electron density variations in the magnetosphere (e.g. Escoubet et al., 1997; Laakso et al., 1997; Johnson et al., 2001).

This paper deals with the distribution of the average electron density in the magnetosphere, using 45 months of satellite potential data from the EFI experiment on the Polar satellite. These data cover $10^{7}$ data points (one-minute averages are used here) distributed between 2 and $9 R_{E}$ geocentric distances along Polar's polar orbit. The plasma regions that directly emerge from the statistical pictures are the cusp, polar cap, and plasmasphere. The data set allows us to investigate the variation of the electron density in these and many other regions. The present study contains a detailed analysis of the polar cap density variation with season, altitude, and geomagnetic activity. We also investigate how the average electron density varies at $L=6.6$, that is, at the geosynchronous distance, as a function of MLT and geomagnetic activity. In the second part of this study, Laakso et al. (2002) present high-resolution data from individual orbits and examine in more detail some specific magnetospheric regions, such as the cusp, plasmapause, trough, and polar cap.

\section{Instrumentation}

We use measurements gathered by the electric field instrument (EFI) on the Polar satellite. Polar was launched on 24 February 1996 into a $90^{\circ}$ inclination orbit with a $9 R_{E}$ apogee (initially over the Northern Hemisphere), a $1.8 R_{E}$

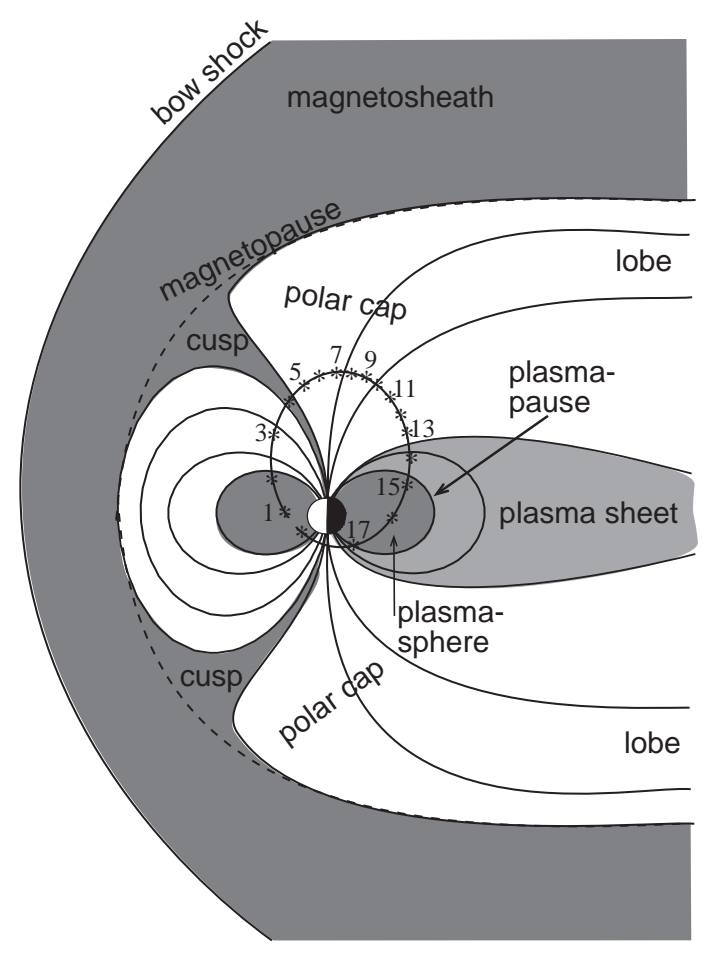

Fig. 1. Schematic drawing of the Earth's magnetosphere in the noon-midnight meridian. A solid line presents a Polar trajectory; asterisks give the spacecraft's position at one-hour intervals.

perigee (initially over the Southern Hemisphere), and an orbital period of about $18 \mathrm{~h}$. The orbital plane rotates about the Earth with respect to the Sun in one year so that all local times are covered in a 6-month period. Figure 1 presents a schematic drawing of the magnetosphere at the noonmidnight meridian and a Polar orbit, where asterisks mark the position of the satellite at one hour intervals.

\subsection{Derivation of total electron density}

The Polar EFI experiment (Harvey et al., 1995) consists of three pairs of double probe antennas oriented perpendicular to each other. The potential difference between each probe (probe potential $\left.=V_{p}\right)$ and the satellite (satellite potential $=$ $\left.V_{s}\right)$ is

$\Delta V_{p s}=V_{p}-V_{s}$,

where $p$ corresponds to the sensor number, and in this case, $p=1-6$. These are sampled at a high-time resolution (10 or $20 \mathrm{~Hz}$, typically), which allows us to follow rapid electron density variations along the Polar trajectory. In burst mode the sampling rate can be up to 8000 samples per second, in which case very fast density variations can be monitored, which are commonly observed, for instance, on auroral field lines (Laakso et al., 1997).

To minimize the contributions of the DC electric fields on the spacecraft potential measurements, the measurements of 


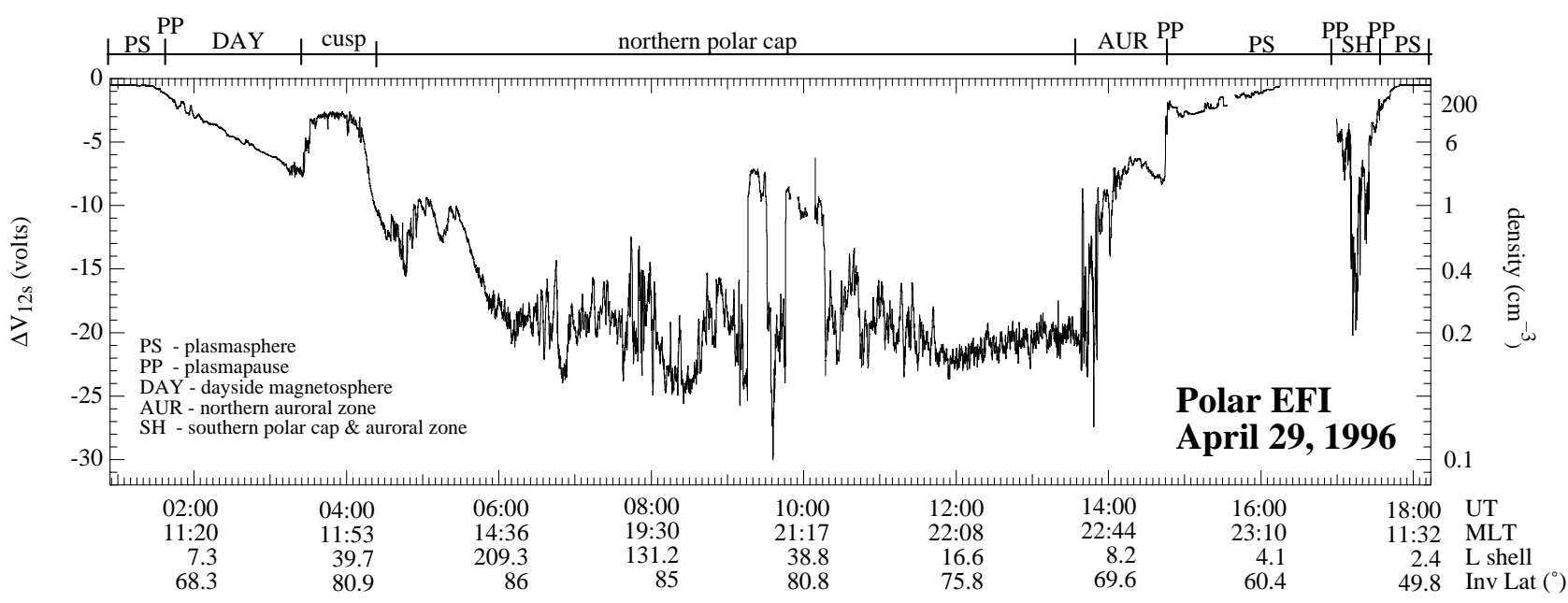

Fig. 2. Negative spacecraft potential $\left(\Delta V_{12 s}\right)$ measurements of the Polar EFI instrument during one orbit on 29 April 1996, when the Polar's orbital plane was near to the midnight-noon meridional plane (for the orbit, see Fig. 1). The right-hand side presents the approximate plasma densities.

the two opposing probes are averaged such that:

$$
\left(\Delta V_{1 s}+\Delta V_{2 s}\right) / 2=\left(V_{1}+V_{2}\right) / 2-V_{s} .
$$

We thus adopt the following notation here: $\Delta V_{12 s}=$ $\left(\Delta V_{1 s}+\Delta V_{2 s}\right) / 2$.

Numerical results show that in a tenuous environment, $\Delta V_{p s}$ and $\log \left(N_{e}\right)$ are related to each other in a way which depends on the temperatures of escaping photoelectrons (Laakso and Pedersen, 1998). Although the magnitude of the saturation photoelectron current is not significant for this relationship, it is essential that the photoelectron current exceeds the sum of the bias current and the ambient electron current. Otherwise, this method of measuring the density fails. In other words, high plasma densities cannot be determined with this technique. The electron temperature $T_{e}$ also affects the value of $\Delta V_{p s}$. However, as shown by Laakso and Pedersen (1998), as long as $T_{e}$ remains in the $1-1000 \mathrm{eV}$ regime, it only gives a minor contribution. The derived and real plasma densities are estimated to differ generally only by less than a factor of 2-3 (mainly due to the differences in $T_{e}$ not taken into account).

The relationship between $\Delta V_{p s}$ and $N_{e}$ can be qualitatively explained as follows: when the density is less than $\sim 500 \mathrm{~cm}^{-3}, V_{s}$ is positive and closely related to the electron density. At the same time $V_{p}$ is kept artificially close to the local plasma potential by means of a bias current which is an electron flux injected from the spacecraft to the probe (Pedersen et al., 1984). Since $V_{p}$ differs from the plasma potential by less than a few volts in the magnetosphere, the measured $\Delta V_{p s}$ is thus very close to $-V_{s}$. When the density increases, $V_{s}$ decreases (and $-V_{s}$ increases) whereas $V_{p}$ remains almost unchanged due to the bias current. Therefore, the factor $\left(V_{1}+V_{2}\right) / 2$ in Eq. (2) is practically constant, and does not present any concern regarding the use of this technique. In high density (above $\sim 500 \mathrm{~cm}^{-3}$ in the Earth's magnetosphere), $\left(V_{1}+V_{2}\right) / 2$ becomes negative, and then the
$\Delta V_{p s}$ measurements can no longer be used as an indicator of the bulk electron density (Laakso and Pedersen, 1998). The actual limit, however, depends on the bias current applied to the sensor and the photoelectron characteristics.

The relationship between $\Delta V_{p s}$ and the total electron density has been empirically studied and modeled recently, using double probe measurements from the GEOS and ISEE satellites (Pedersen, 1995; Escoubet et al., 1997). Using 10 months of Polar data from the EFI and HYDRA instruments, Scudder et al. (2000) performed a detailed investigation of this relationship. In fact, the present study utilizes their derived relationship, which is quite similar to the relationships found for other satellites (Pedersen, 1995).

\subsection{Variation of $\Delta V_{12 s}$ along Polar's orbit}

Figure 2 shows an example of the $\Delta V_{12 s}$ variation along one full orbit on 29 April 1996, when Polar's orbital plane was near the noon-midnight meridian (see Fig. 1); the density scale is given on the right-hand side. Notice that the highdensity regions appear as weakly negative $\Delta V_{12 s}$ values. In the plasmasphere $\Delta V_{12 s}$ is more than $-2 \mathrm{~V}$, but during the outbound crossing of the plasmapause it rapidly decreases by several volts. In this voltage range, such a small change corresponds to a large density decline by a few orders of magnitude. The characteristics of the density gradient at the plasmapause can, in general, vary quite significantly. For instance, in Fig. 2 the plasmapause appears very clearly at about 14:45 UT near midnight, but less clearly at 01:45 UT near noon. This point with other examples is investigated in more detail in the companion paper (Laakso et al., 2002).

An interesting feature in Fig. 2 is the crossing of the cusp at 03:30-04:15 UT, during which $\Delta V_{12 s}$ increases from $-7 \mathrm{~V}$ to $-2.5 \mathrm{~V}$. This corresponds to a density increase from 2 to $50 \mathrm{~cm}^{-3}$. Marklund et al. (1990) observed similar variations across the cusp with the Viking satellite at somewhat 
lower altitudes. Poleward of the cusp, centred at 05:00 UT, another density enhancement occurs, which is possibly related to the inner edge of the high-latitude boundary layer or the plasma mantle (Rosenbauer et al., 1975).

In the polar cap regions, which map into the lobes of the magnetotail, $\Delta V_{12 s}$ becomes strongly negative. In Fig. 2, $\Delta V_{12 s}$ decreases to $-25 \mathrm{~V}$ (which corresponds to densities below $\sim 0.1 \mathrm{~cm}^{-3}$ ) (Scudder et al., 2000). Quite frequently, the potential in the polar cap is between -30 and $-40 \mathrm{~V}$ and can sometimes go below $-50 \mathrm{~V}$ (i.e. the density is near $0.01 \mathrm{~cm}^{-3}$ ). Figure 2 also shows large-scale density structures in the high-altitude polar cap region. Such variations are commonly observed, and are likely a mixture of temporal and spatial structures, related to solar wind-magnetosphereionosphere coupling processes. Over the Southern Hemisphere the satellite crosses the polar cap very quickly (17:2017:30 UT). Here the satellite's altitude is only $6000 \mathrm{~km}$ or less, and $\Delta V_{12 s}$ varies between -5 and $-12 \mathrm{~V}$, corresponding to a density range $0.8-2 \mathrm{~cm}^{-3}$. Just prior to this, the spacecraft is above the southern auroral oval, where $\Delta V_{12 s}$ is below $-20 \mathrm{~V}$ due to a low-altitude auroral cavity (corresponding to a density of $0.2 \mathrm{~cm}^{-3}$ ).

\section{Statistical results}

We now use spacecraft potential measurements from 1 April 1996 to 31 December 1999, to provide average density distributions within the magnetosphere. Since the magnetospheric structure strongly depends on the geomagnetic activity, the measurements are binned by 3-hour $K_{p}$ index.

Before the spacecraft potential data are used in the statistical analysis, intervals with poor data quality must be removed. Such intervals result from a variety of reasons. First, the Polar satellite carries a satellite potential control device, called PSI (Moore et al., 1995), which is used at selected intervals to keep the satellite at a constant low potential, whatever the ambient plasma density. Therefore, all intervals of PSI operation have been deleted from this study. Second, the EFI pre-amplifiers oscillate during times of high electron fluxes (F. Mozer, private communication, 1996), and the data is of very poor quality for those occasions. This may happen sometimes in the magnetosheath and often in the highdensity plasmasphere (within about $\mathrm{L}=3-3.5$ ). The events of the first type have not been removed as their appearance is difficult to discern automatically; fortunately, they occur so seldom that they have no influence on the average picture. The second case is more serious, and the corresponding intervals have been removed using an empirical relationship that compares the spacecraft potential on L-shells less than 3.5; if the spacecraft potential is larger than $2 \mathrm{~V}$ in that region, it is likely due to probe oscillation, and the corresponding interval has been removed. Since this happens only in the highdensity environment (more than $\sim 500 \mathrm{~cm}^{-3}$ ), it has only minor effects on the results of this study which is focussed primarily on density patterns near or outside the plasmapause. Third, the electric field instrument frequently performs on- board calibrations by sweeping the bias currents, which directly affects the spacecraft potential. These intervals have also been deleted from the data set.

\subsection{Meridional electron density distributions}

Average electron density distributions are displayed in two meridional planes in Fig. 3a. The left panels are for the noonmidnight meridian, and the right panels are for the duskdawn meridian; the azimuthal extent of the sectors is $\pm 1 \mathrm{~h}$. The panels from top to bottom are for three different $K_{p}$ levels: $K_{p}=0-1^{-}$(top panels), $K_{p}=1^{+}-2$ (middle panels), and $K_{p} \geq 3^{-}$(bottom panels), which correspond to magnetically quiet, moderately active, and disturbed periods, respectively. The lines in each panel represent the dipole field lines at $65^{\circ}, 70^{\circ}, 75^{\circ}$, and $80^{\circ}$ invariant latitude, or 5.6, 8.5, 14.9, and 32.3 L-shell. The color scale shown at the top of the figure is logarithmic and ranges from 0.2 to $100 \mathrm{~cm}^{-3}$.

The selected three $K_{p}$ ranges have approximately an equal amount of data (the number of data points, each of which is a one-minute average, are approximately 424000,535000 , and 539000 for $K_{p}=0-1^{-}, K_{p}=1^{+}-2$, and $K_{p} \geq 3^{-}$, respectively). Similar displays showing the count distributions are presented in Fig. 3b. Since the number of active days is small, we have binned all of the data gathered for $K_{p} \geq 3^{-}$ together. Most bins contain several tens of counts and each count is an average of about $200 \Delta V_{p s}$ measurements for one minute so that the statistics of Fig. 3a can be considered satisfactory.

When interpreting the results of Fig. 3a, one must notice that on some occasions the average density may not be a representative value. For instance, in a bin which is frequently near the plasmapause, one may detect densities from $0.1 \mathrm{~cm}^{-3}$ to a few $100 \mathrm{~cm}^{-3}$. An average density for this bin may well be something near $10 \mathrm{~cm}^{-3}$, which does not represent a typical density, since this concept is somewhat ill-defined in such cases (for more detail, see Sect. 4.3).

In the left panels of Fig. 3a, the cusp appears as a relatively dense plasma region near $80-82^{\circ}$ of invariant latitude near noon, and it tends to move equatorward with increasing $K_{p}$. The average density in the cusp is a few electrons per $\mathrm{cm}^{3}$, which is less than the average magnetosheath density. Examination of individual cusp crossings (see Laakso et al., 2002) reveal that the cusp densities are often several $10 \mathrm{~cm}^{-3}$, whereas the densities in the adjacent boundary layers are one or two orders of magnitude less. All bins near the cusp region contain values from the cusp and boundary layers, resulting in averages that are not more than a few electrons per $\mathrm{cm}^{3}$. Another reason for low average cusp densities is the motion of the cusp away from the noon sector during large IMF $\mathrm{B}_{y}$ values. Although the cusp certainly exists at all $K_{p}$ levels, it appears less distinctly in these average bins during disturbed intervals. The large variation in the cusp position during disturbed conditions likely spreads out the cusp signatures in the average picture (see also Palmroth et al., 2001). 


\section{Polar EFI: 1 April 199631 Dec 1999}
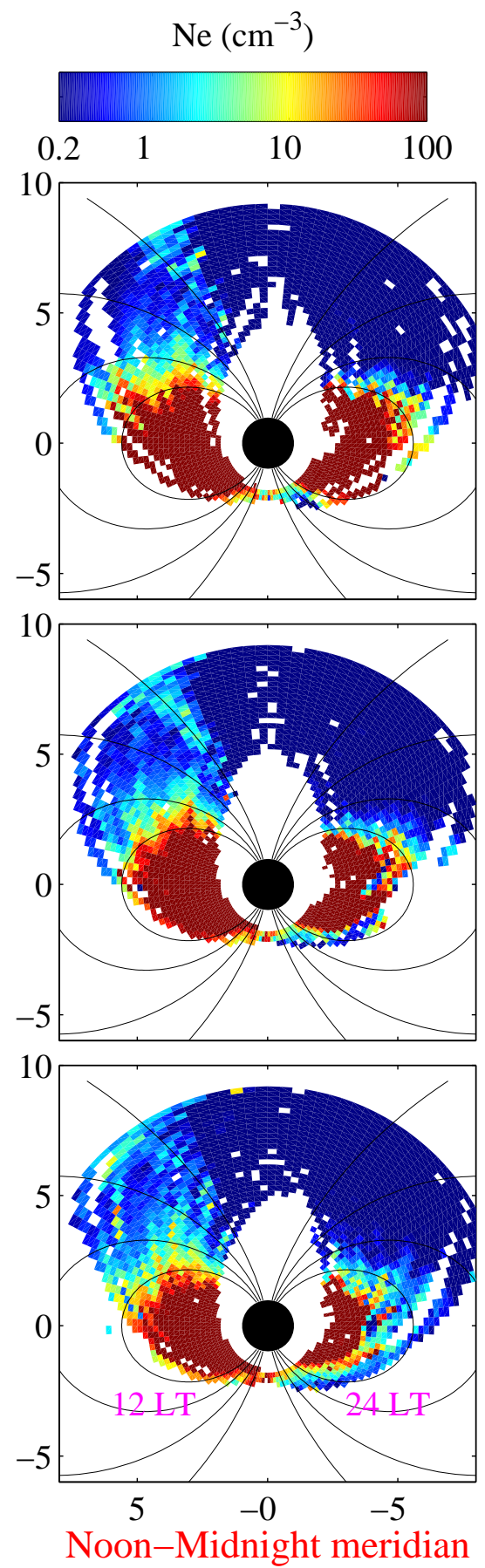
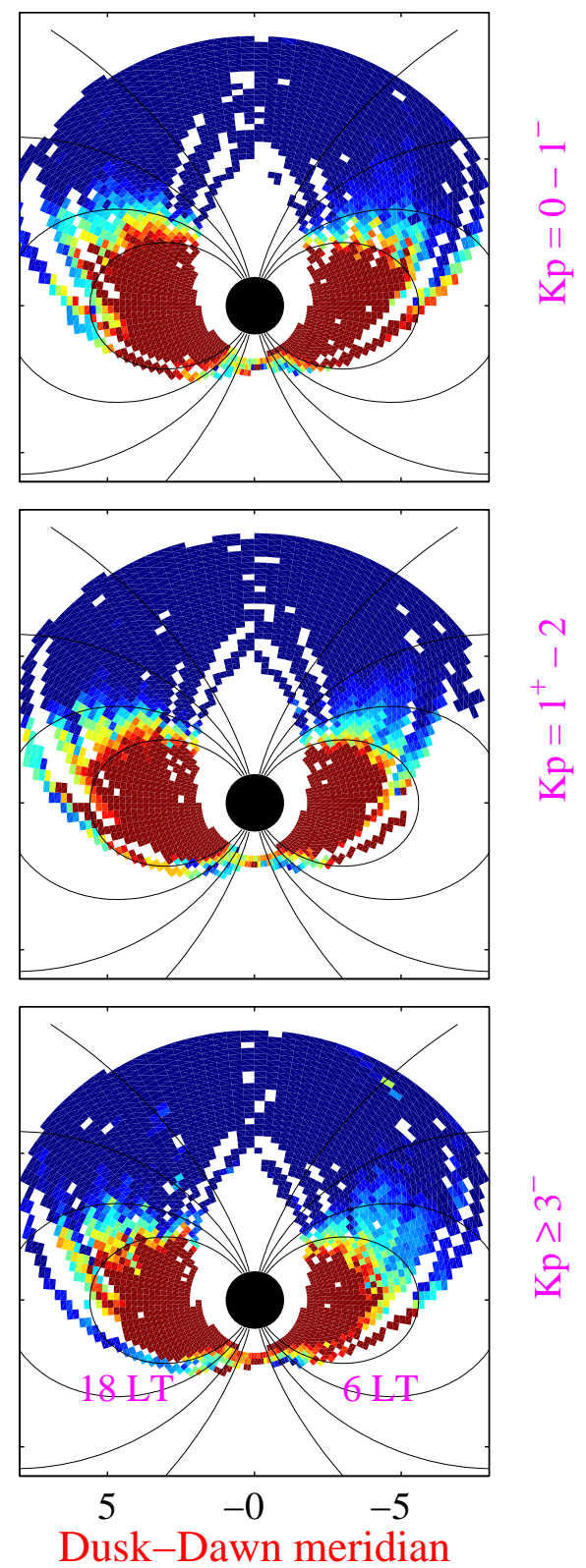

Fig. 3a. (a) Average electron density in two meridional planes (scales in $R_{E}$ ): left panels are for the noon-midnight meridian, and right panels are for the dusk-dawn meridian. The panels from top to bottom are for three different $K_{p}$ levels: $K_{p}=0-1^{-}, K_{p}=1^{+}-2$ and $K_{p} \geq 3^{-}$. The lines in each panel represent dipole magnetic field lines at invariant latitudes of $65^{\circ}, 70^{\circ}, 75^{\circ}$, and $80^{\circ}$. (b) Number of counts used for the calculation of the average densities.
The polar cap region, which maps into the lobes of the magnetotail, is very tenuous and appears as dark blue in Fig. 3a. The average densities are around $0.2 \mathrm{~cm}^{-3}$ or less above $4 R_{E}$. It is obvious that there is a significant altitude dependence here, since the average density is more than an order of magnitude higher at $1 R_{E}$ altitude (southern polar cap) than at $4-8 R_{E}$ altitude (northern polar cap). This is discussed in more detail in Sect. 4.2. In addition, as will be discussed in Sect. 4.1, the seasonal dependence is very strong and dominates the density variation in the polar cap at any altitude. Notice that in the left-hand panels the southern polar cap density is asymmetric about the magnetic pole so that lower densities are observed in the nighttime sector. This is because the Sun is not illuminating the nightside ionosphere 


\section{Polar EFI: 1 April 199631 Dec 1999}
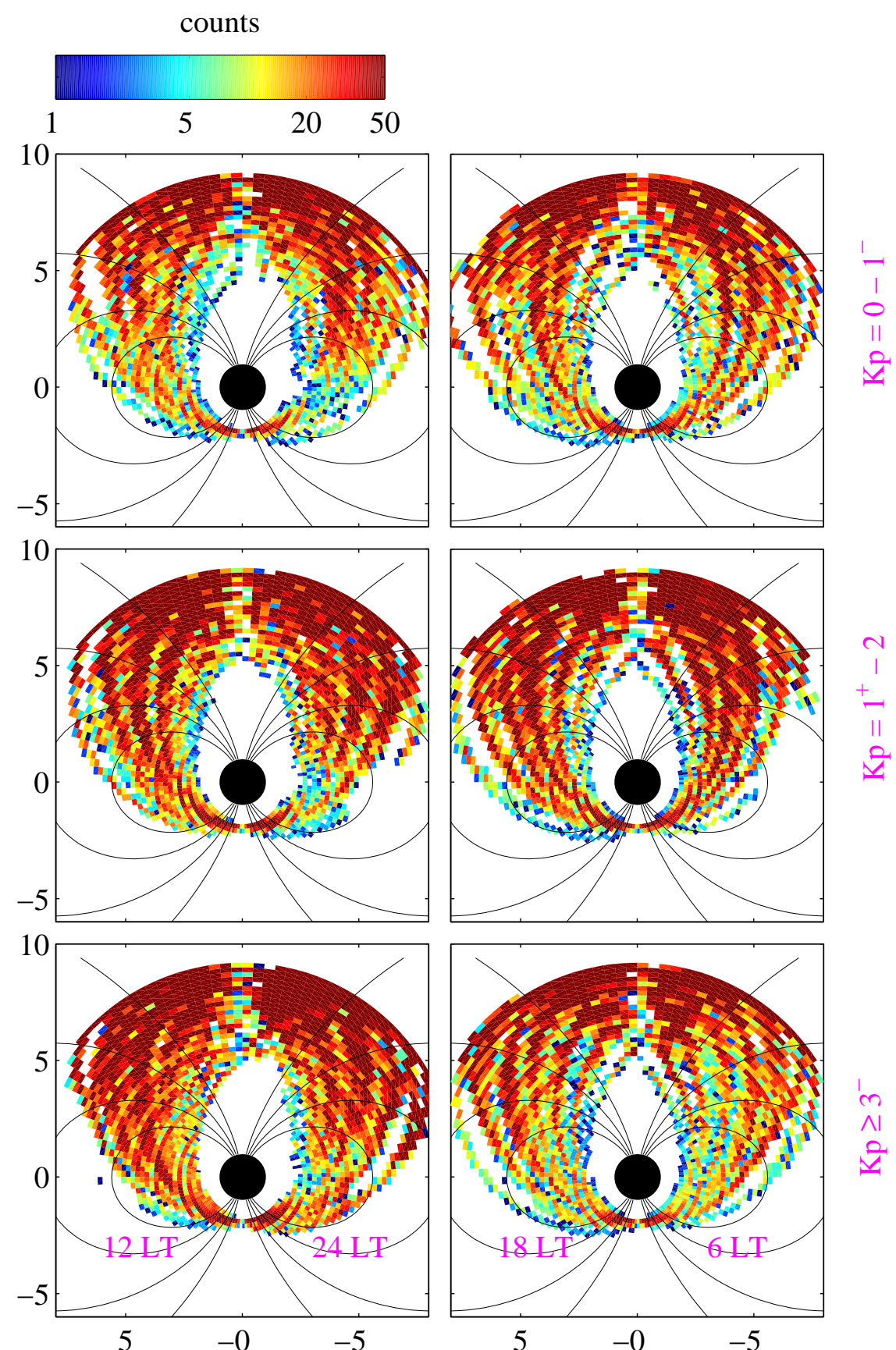

Noon-Midnight meridian

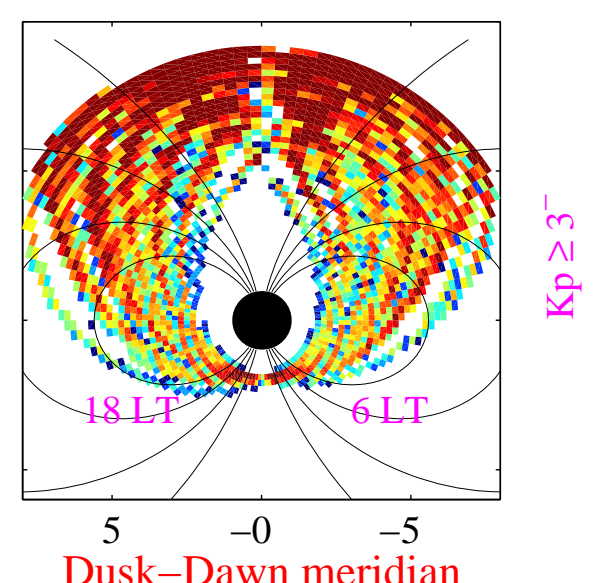

Fig. $\mathbf{3 b}$. continued ....

in the polar cap. Finally, it seems that the low-altitude (southern) polar cap density may increase with $K_{p}$; this will also be investigated in more quantitative detail in Sect. 4.1.

When average plasma densities for the same L-shells are compared between the dayside and nightside, it seems as if the densities are higher on the dayside than on the nightside. In particular, the average densities between the plasmapause and the magnetopause (between $65^{\circ}$ and $75^{\circ}$ in invariant latitude) can be quite high, up to $10 \mathrm{~cm}^{-3}$ on the dayside and dawnside, whereas in the dusk and midnight sectors the average densities on the same L-shells are lower. This is investigated in more detail in the companion paper (Laakso et al., 2002).

In Fig. 3a, the average plasmapause location appears ap- 
Polar satellite: 1 Apr 199631 Dec 1999
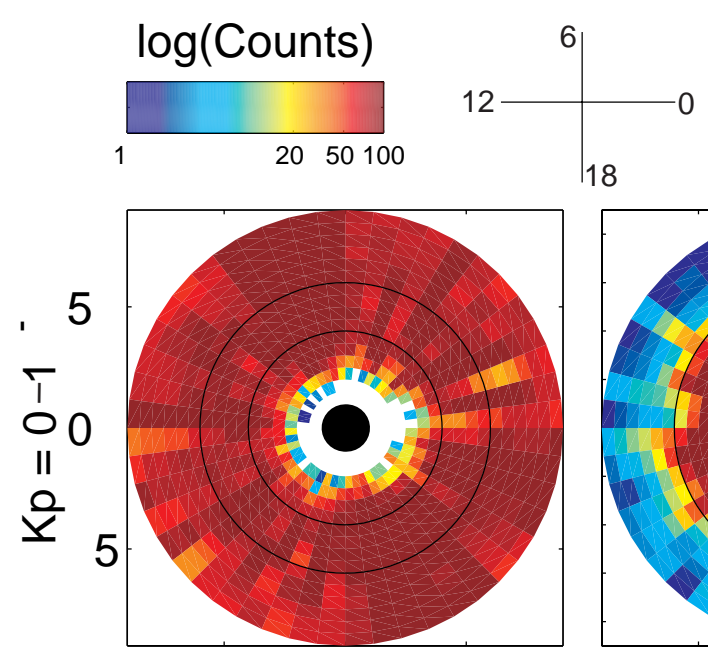

$\log \left(\mathrm{N}_{\mathrm{e}}\right)$ per cc
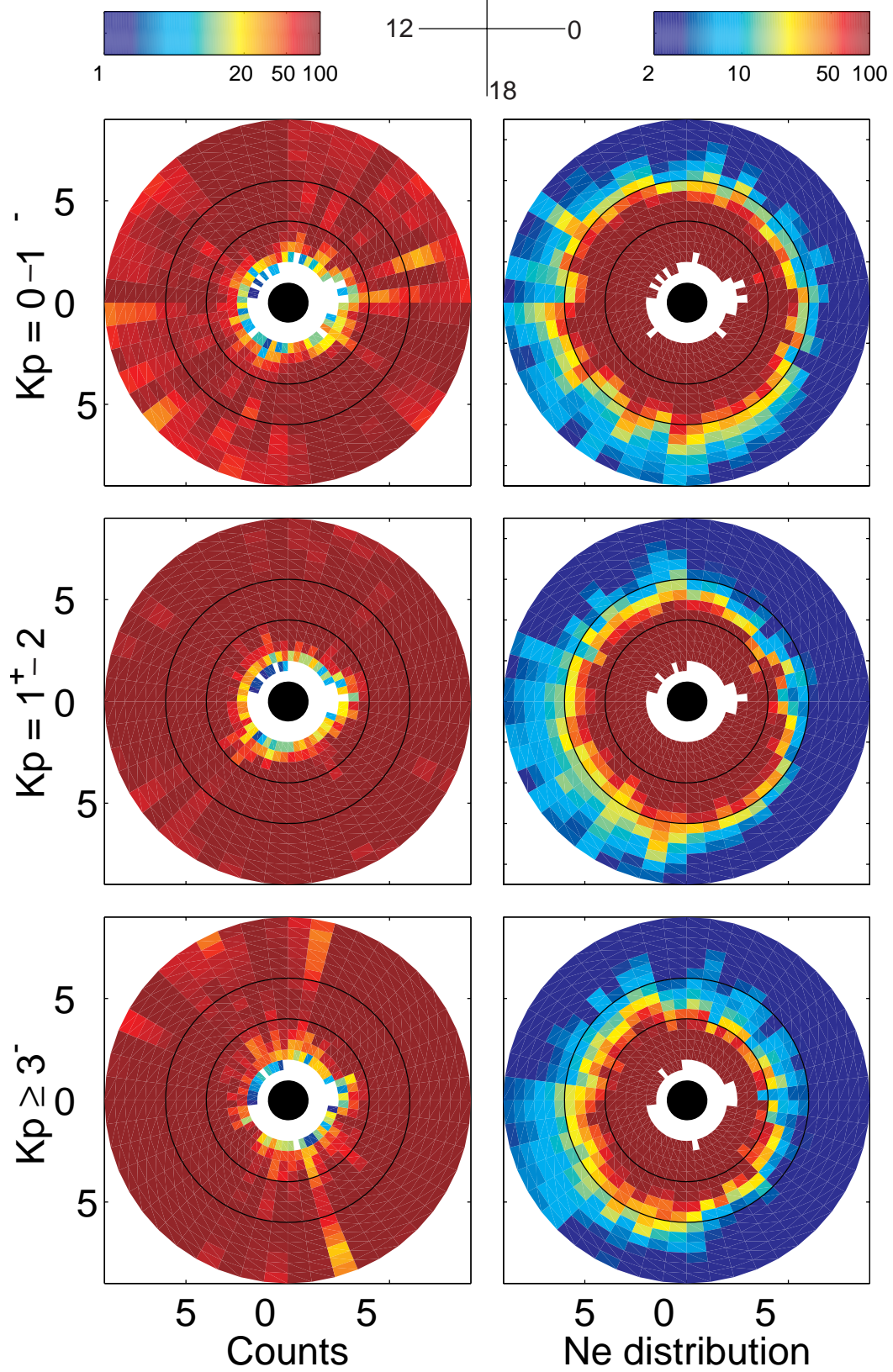
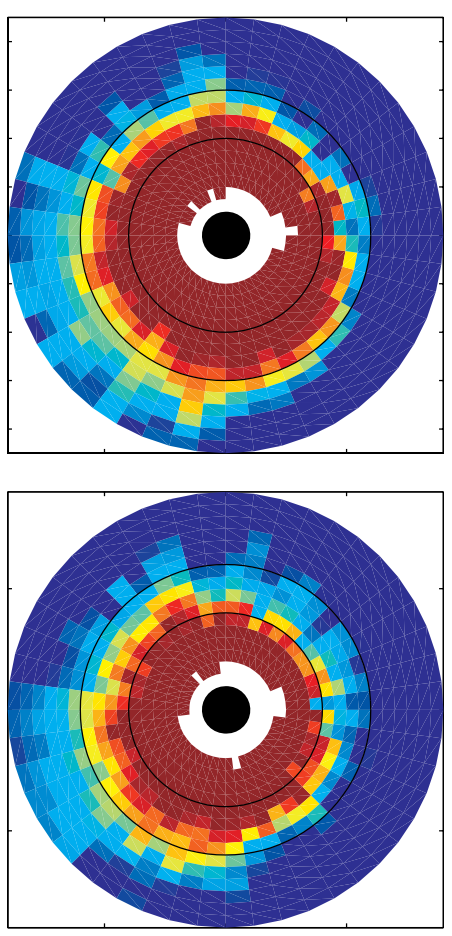

$\begin{array}{lll}5 & 0 & 5\end{array}$

Ne distribution
Fig. 4. Right panels: Average electron density distributions (scales in $R_{E}$ ) in the inner magnetosphere mapped to the magnetic equator (i.e. within $\mathrm{L}=9$ ). The panels from top to bottom represent three different $K_{p}$ levels (same as in Fig. 3). The circles in each panel show the equatorial distances of $L=4$ and 6. Left panels: Number of counts used for the calculation of the average densities. proximately at the edge of a dark red color which corresponds to a density of about $100 \mathrm{~cm}^{-3}$. Not only is the density decline at the plasmapause sharpest near midnight, but also the plasmapause location correlates most clearly with $K_{p}$ at midnight and dawn, implying that the dependence of the earthward motion of the plasmapause on $K_{p}$ is strongest. The right-hand panels in Fig. 3a show a clear asymmetry between the dawnside and duskside plasmapause, as shown by numerous previous studies (e.g. Carpenter et al., 1993; Lemaire and Gringauz, 1998; Laakso and Jarva, 2001). The statistical results presented here demonstrate that the asymmetry increases with increasing $K_{p}$, presumably due to strong motion of the dawnside plasmapause.

\subsection{Equatorial electron density distributions}

The right-hand panels of Fig. 4 present the average electron densities which have been mapped to the magnetic equator within the inner magnetosphere to $L=9$; the number of counts in each bin is shown in the left panels. Our knowledge about the electron density distribution along a magnetic field line is quite poor and thus, the mapping of the density along 
a field line is difficult to perform in general. However, over the Northern Hemisphere for $L \leq 9$, the satellite is always located at high altitudes and low magnetic latitudes, being quite close to the magnetic equator; for instance, the $\mathrm{L}=9$ field line is crossed at $40^{\circ}$ or less of magnetic latitude over the Northern Hemisphere. For comparison, over the Southern Hemisphere, the plasmapause is always crossed at low altitudes and high magnetic latitudes (see Fig. 1). For an incompressible plasma, the density should be proportional to the cross section of the flux tube in which the plasma is located. For the magnetic latitude range $0-40^{\circ}$ at $\mathrm{L}=4-9$, this cross section does not change much, although for detailed modeling analysis it should be taken into account. Since the density is thus not expected to change very much between the measurement point and the equator, the electron density may be assumed constant along the magnetic field lines. However, only measurements from the Northern Hemisphere are used here in order to avoid problems in mapping from the Southern Hemisphere.

The mapping is based on a dipole field model, which is acceptable in the inner magnetosphere, although this assumption is less reliable during disturbed conditions. As in Fig. 3a, the panels from top to bottom in Fig. 4 are for three different $K_{p}$ levels: $K_{p}=0-1^{-}, K_{p}=1^{+}-2$, and $K_{p} \geq 3^{-}$. In each panel, the circles are at 4 and $6 R_{E}$, the Sun is to the left, the magnetotail to the right, the dawnside upward and the duskside downward. The color scale is logarithmic, as in Fig. 3a, representing densities ranging from 0.2 to $100 \mathrm{~cm}^{-3}$.

The data in Fig. 4 suggests that the steady-state plasmapause moves earthward with increasing $K_{p}$. This effect is most pronounced on the nightside and is likely due to the effect of the increasing convection electric field during disturbed conditions. The duskside plasmaspheric bulge, discussed by several authors (Carpenter et al., 1993; Gallagher et al., 1995) and caused by the cancellation of corotational electric fields by convection electric fields (Lyons and Williams, 1984; Lemaire and Gringauz, 1998), is particularly clear for high $K_{p}$. An interesting feature in Fig. 4 is the appearance of a dense region pointing from the plasmasphere toward the magnetopause in the 12-15 MLT sector, which may be related to detached plasmaspheric material that is transported toward the dayside magnetopause (Kurita and Hasegawa, 1985).

One must bear in mind that the plasmasphere can experience drastic changes during disturbed intervals, and in these cases the statistical pictures, such as those shown in Fig. 4, do not represent the plasmasphere well at all. For instance, Fig. 4 suggests that the position of the duskside plasmapause does not change much with $K_{p}$. However, from previous studies (Carpenter et al., 1993; Gallagher et al., 1995) we know that the plasmaspheric bulge is a very dynamic region, with strong erosion during high geomagnetic activity. Thus, this is an example of how average distributions or patterns, such as that shown in of Figs. 3a and 4, can be misleading if used for studying the behavior of dynamic regions, such as the plasmaspheric bulge.

\section{Discussion}

We have used forty-five months of spacecraft potential measurements collected by the electric field double probe on the Polar satellite to reveal electron density variations in the magnetosphere and to show how these depend on geomagnetic activity and season. Even though the observations are binned by $K_{p}$, the highly averaged density distributions yield static pictures, and the conclusions made from such plots must be taken with caution. Therefore, observations from individual orbits have been used to check the validity of the average patterns; the results are presented by Laakso et al. (2002) in the second part of this study.

Next we will discuss a few issues in more detail from a statistical point of view. First, Fig. 3a suggests that the polar cap plasma density varies with geomagnetic activity, and, therefore, in Sect. 4.1, we examine both the seasonal and geomagnetic dependence of the polar cap density. Second, also according to Fig. 3a, the polar cap density appears to be quite different at low (southern) and high (northern) altitudes. Combining the measurements from both hemispheres, the data points provide good altitude coverage, and we present a simple model on the altitude variation of the electron density in Sect. 4.2. Finally, based upon the data set of Fig. 4, we can investigate the local time variation of the average density at any distance or L-shell. In Sect. 4.3 we particularly concentrate on the local time variation of the average density at the geosynchronous distance under different geomagnetic conditions.

\subsection{Seasonal variation of the polar cap density}

The polar cap plasma density is affected primarily by the Sun illuminating the polar ionosphere, which directly causes a significant seasonal variation of the polar cap density. We now investigate this variation by averaging all observations when the invarant latitude of the satellite is greater than $82^{\circ}$ (or L > 50) for both the Southern Hemisphere and the Northern Hemisphere; this criterion was adopted in order to avoid the contributions from the cusp and the auroral oval on the average densities. We have also binned the data into three $K_{p}$ ranges: $K_{p}=0-1$ (quiet periods), $K_{p}=1^{+}-2^{+}$(moderate disturbances), and $K_{p} \geq 4^{-}$(strong disturbances), in order to separate the effect of geomagnetic activity on the polar cap density. The results are presented in Fig. 5. The upper panel is for the northern, high-altitude polar cap, and the lower panel is for the southern, low-altitude polar cap. An upper limit of the density of $300 \mathrm{~cm}^{-3}$ was taken for the calculation of the average. For the low-altitude summer polar cap, where very high densities, exceeding $300 \mathrm{~cm}^{-3}$, can occur (Laakso et al., 2002), the values shown in Fig. 5 can, therefore, be somewhat underestimated. This figure shows that at small distances $\left(\sim 2 R_{E}\right)$, the average density varies between $1 \mathrm{~cm}^{-3}$ for dark conditions and $100 \mathrm{~cm}^{-3}$ for sunlit conditions, whereas at higher distances $\left(5-9 R_{E}\right)$ the amplitude of the density variation between the two regions is typically much less, being in the range of $0.03-0.3 \mathrm{~cm}^{-3}$. So, 

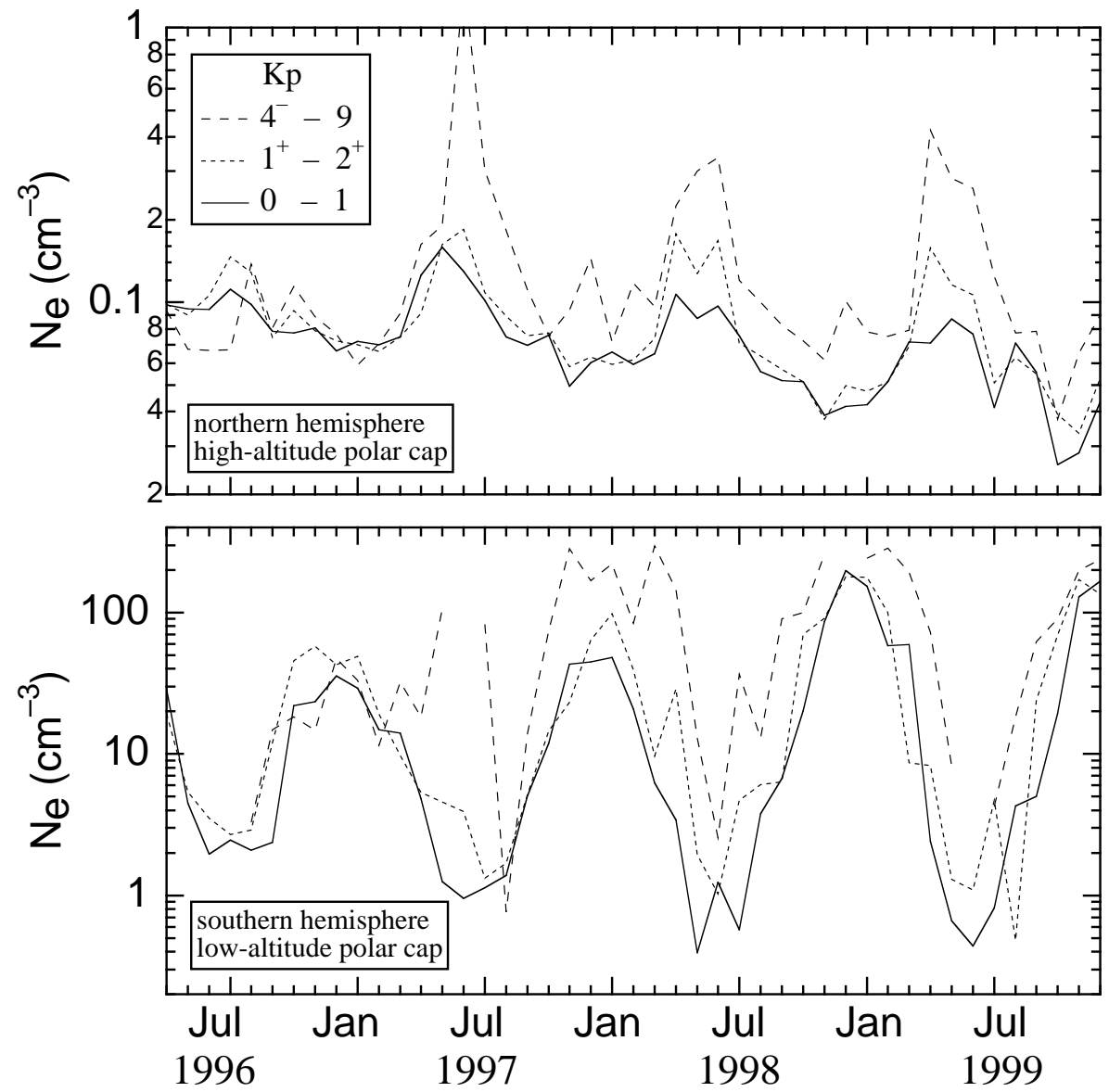

Fig. 5. The monthly averages of the electron density in the polar cap region over the Southern Hemisphere (upper panel) and the Northern Hemisphere (lower panel). The measurements in different geomagnetic conditions are separated: solid lines are for quiet conditions, dotted lines for moderately active conditions, and dashed lines for disturbed conditions. Note the different density scales between the two panels. the low-altitude polar cap density increases by one or two orders of magnitude from winter to summer, whereas the highaltitude density increases only by a factor of $2-10$ between these seasons.

These densities are in the same range as those observed by Persoon et al. (1983) using DE-1 measurements. They obtained an average density of $30 \mathrm{~cm}^{-3}$ at $2 R_{E}$ distance and $1 \mathrm{~cm}^{-3}$ at $4.5 R_{E}$ distance; however, they averaged all data at $\mathrm{L}$ greater than 10 ( $71^{\circ}$ of invariant latitude) from September to February over the Northern Hemisphere.

Geomagnetic activity also affects the polar cap density. In summer, the average density in the low-altitude polar cap increases by a factor of 2-10 during high $K_{p}$, while in winter months (May-August, for the Southern Hemisphere) the density may increase one or two orders of magnitude. Of course, for an individual event, the density enhancement can even be much more dramatic. At high altitudes (northern polar cap data), the variation of the average density with magnetic activity is also clear, with density increases of a factor of 2-5 during high $K_{p}$, but this factor appears to be quite independent of season, contrary to low-altitude observations. We speculate that during high $K_{p}$ the low-altitude polar cap is readily filled by heavy ionospheric ions that are gravitationally bound and cannot escape to the high altitudes.

\subsection{Altitude variation of the polar cap density}

To study the altitude variation of the polar cap density, the measurements must be collected during the same season and also under similar geomagnetic conditions. For example, when comparing similar seasons and magnetic activity periods shown in Fig. 5, it is clear that in the winter the density decreases by a factor of 10-20 from low to high altitudes, whereas in the summer the decline is typically much steeper, of the order of 200-1000.

We now investigate one case in more detail. Figure 6 presents the polar cap density versus altitude for dark conditions (December-January for the Northern Hemisphere, and June-July for the southern one) during very quiet intervals $\left(K_{p}=0-0^{+}\right)$. In order to decrease the scatter of data points, the measurements over the Northern Hemisphere are averaged over $20 \mathrm{~min}$, whereas the data from the Southern Hemisphere near $2 R_{E}$ are one-minute averages. The figure presents two reference lines: the dashed line, proportional to distance as $r^{-3}$, represents the field-aligned outflow with a constant flow velocity, whereas the solid line, $r^{-4.4}$, shows the best fit to the data points below $7 R_{E}$ distance; for reference, using DE-1 data, Persoon et al. (1983) obtained a radial dependence of $r^{-3.8}$. Thus, the Polar data suggest that the plasma particles are accelerated as $r^{1.4}$ with distance, which 

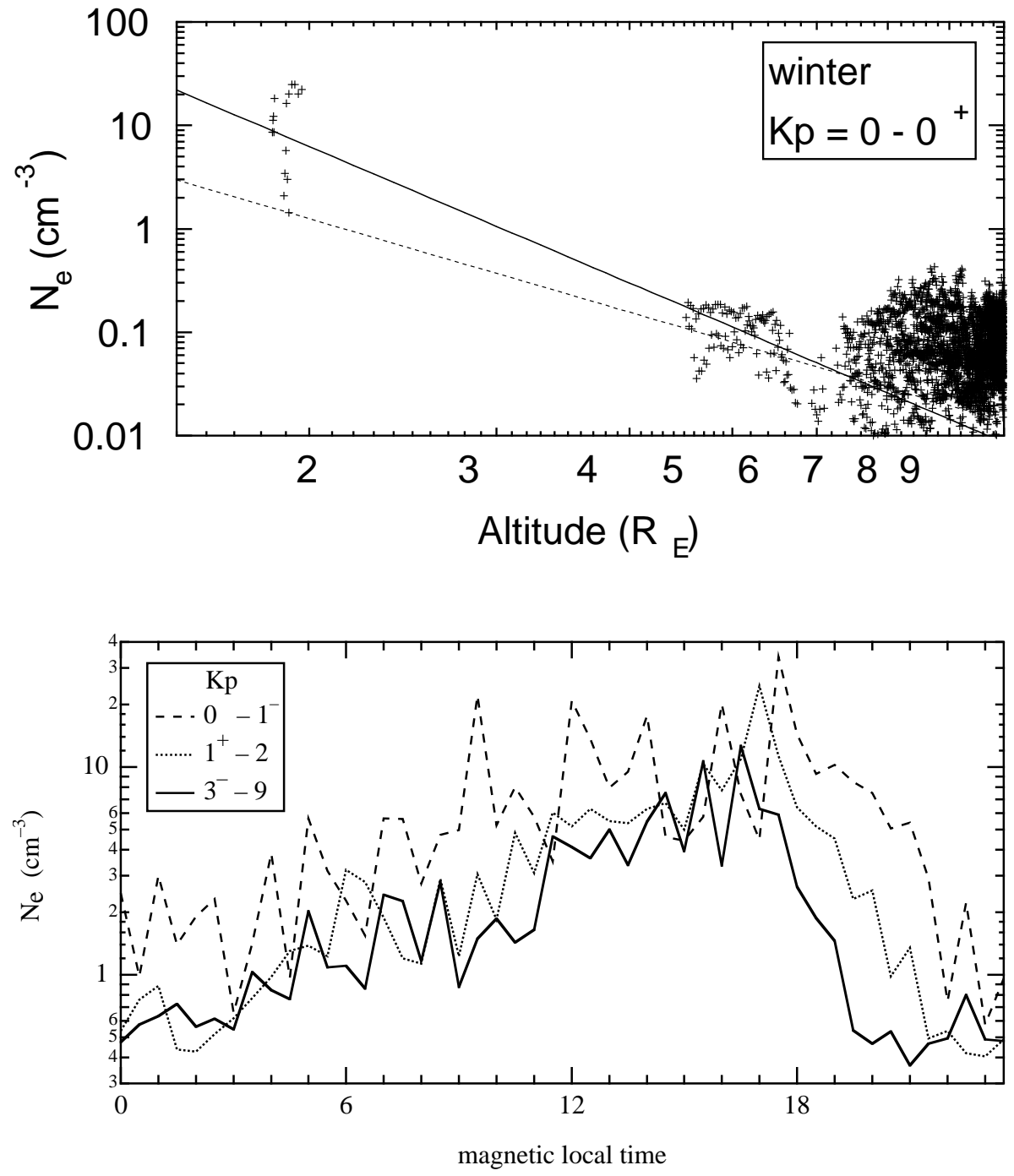

Fig. 6. Polar cap density against altitude for dark hemispheres during very quiet intervals $\left(K_{p}=0-0^{+}\right)$. The dashed line, proportional to distance as $r^{-3}$, represents the radial outflow with constant flow velocity, and the solid line, $r^{-4.4}$, is the best fit to the data points below $7 R_{E}$ distance.

Fig. 7. Average electron densities at $\mathrm{L}=6.6$ plotted against magnetic local time, based on 45 months of Polar data over the Northern Hemisphere. Densities are binned with the $K_{p}$ index as in Fig. 4.

is somewhat faster than observed by DE-1. When observations during higher $K_{p}$ ranges are examined, a similar result is obtained. A more complete analysis on this subject is presented by Laakso and Grard (2002).

Above $7 R_{E}$, the data points seem to form a flat distribution, likely because the magnetic field model is not dipolar at high altitudes but also because at these distances the spacecraft may be immersed in the high-latitude boundary layer and the cusp rather than in the polar cap. This is in accordance with the observations of Palmroth et al. (2001), which indicate that above $7 R_{E}$ distance the cusp may spread out over a large spatial volume.

\subsection{Plasma distributions at geosynchronous distance $(\mathrm{L}=6.6)$}

Using the average densities of Fig. 4, we now study the local time variation of the average density near $\mathrm{L}=6.6$, which corresponds to the geosynchronous distance at the equator. The results are shown in Fig. 7 where the dashed line gives the average density for the quiet periods, the dotted line for the moderate disturbances, and the solid line for the disturbed conditions. This figure shows that the density at geosynchronous orbit tends to decrease with increasing $K_{p}$ at all sectors except the bulge sector (14-17 MLT). Particularly large density changes appear in the pre-midnight sector, where the density gradient from 18 MLT to 23 MLT becomes steeper with increasing $K_{p}$ and moves sunward. This is the region that is most strongly affected by geomagnetic disturbances. In the post-midnight sector, the densities can also vary significantly, due to a strong radial motion of the plasmapause (see Fig. 4). Large density variations in this sector can occur particularly during quiet periods, which can be associated with the expansion of the plasmasphere, with the detachments of plasmaspheric plasma (Chappell, 1974), or with plume-type plasmaspheric structures (Ober et al., 1997). The interpretation of the average values shown in Fig. 4 must be carried out with caution since the average values are not necessarily well-defined variables in a region where the density can have a wide range of values. This applies particularly to the geo- 

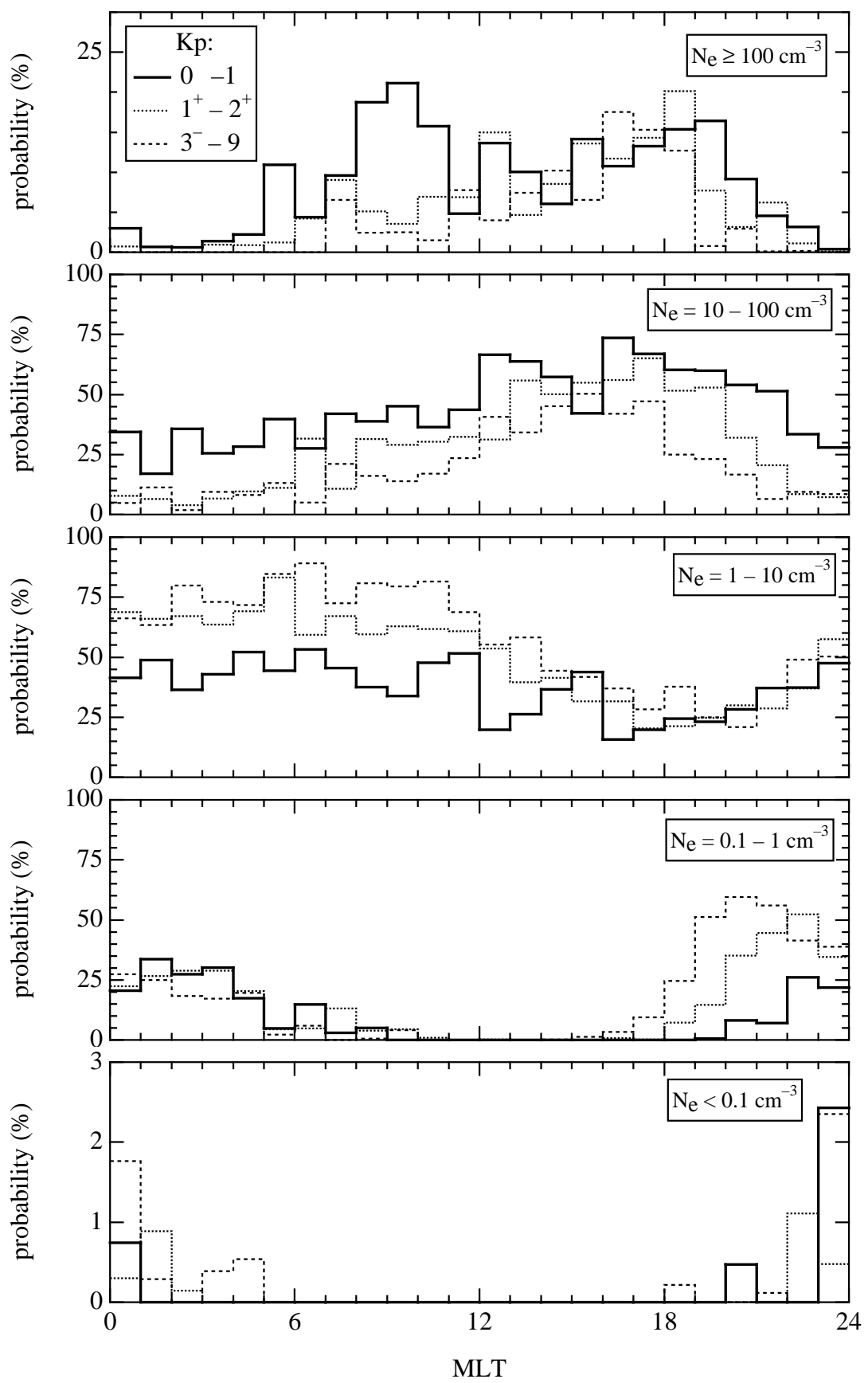

Fig. 8. Probabilities of detecting different density regimes at the geosynchronous distance as function of MLT. Three $K_{p}$ ranges are used: quiet periods (solid lines), weak disturbances (dotted lines), and strong disturbances (dashed lines). This figure uses data of Fig. 4.

stationary orbit that can often be in the plasmasphere, trough, or plasma sheet.

The data in Fig. 7 clearly shows that the average density increases an order of magnitude from the nightside to the dayside: the average density varies in the range of $0.6-$ $30 \mathrm{~cm}^{-3}$ for quiet intervals and in the range of $0.4-10 \mathrm{~cm}^{-3}$ for disturbed intervals; the low values correspond to a typical trough density and the high values to a typical plasmaspheric density at $\mathrm{L}=6.6$. As discussed above, the average values may not always be well-defined variables in this region, but nevertheless these results agree surprisingly well with daily variations reported by Higel and Wu (1984) (see their Fig. 2).

We continue our investigation by showing the probabilities of detecting different density regimes at the geosynchronous distance as a function of magnetic local time and $K_{p}$ (Fig. 8). Very low densities (i.e. below $0.1 \mathrm{~cm}^{-3}$ ), possibly representing the encounters of auroral cavities or the lobes, are observed at geostationary orbit approximately $2 \%$ of the time around local midnight, but hardly ever in the 0518 MLT sector. Due to a low number of events, a possible 
Table 1a. Occurrence probabilities (\%) of plasma regions at $\mathrm{L}=6.6$ for $K_{p}=0-1$

\begin{tabular}{lrrrr}
\hline MLT: & $00-06$ & $06-12$ & $12-18$ & $18-24$ \\
\hline plasma sheet & 23 & 4 & 0 & 11 \\
trough & 44 & 45 & 27 & 33 \\
plasmasphere (normal) & 30 & 39 & 62 & 48 \\
plasmasphere (dense) & 3 & 12 & 11 & 8 \\
\hline
\end{tabular}

Table 1b. As Table 1a, but for $K_{p}=1^{+}-2^{+}$

\begin{tabular}{lrrrr}
\hline MLT: & $00-06$ & $06-12$ & $12-18$ & $18-24$ \\
\hline plasma sheet & 22 & 4 & 0 & 31 \\
trough & 70 & 62 & 36 & 33 \\
plasmasphere (normal) & 7 & 28 & 52 & 29 \\
plasmasphere (dense) & 1 & 6 & 12 & 7 \\
\hline
\end{tabular}

Table 1c. As Table 1a, but for $K_{p}=3-9$

\begin{tabular}{lrrrr}
\hline MLT: & $00-06$ & $06-12$ & $12-18$ & $18-24$ \\
\hline plasma sheet & 19 & 2 & 3 & 45 \\
trough & 73 & 79 & 44 & 37 \\
plasmasphere (normal) & 8 & 16 & 43 & 15 \\
plasmasphere (dense) & 0 & 3 & 10 & 3 \\
\hline
\end{tabular}

$K_{p}$ dependence cannot be resolved. The results for the other density regimes are summarized in Tables $1 \mathrm{a}-\mathrm{c}$ for four MLT sectors. Based on the local time occurrence of the observed density distributions, we can divide the measurements into the following general groups: the density range $>100 \mathrm{~cm}^{-3}$ represents the dense plasmasphere (saturated flux tubes), the range $10-100 \mathrm{~cm}^{-3}$ represents the normal plasmasphere, the range $1-10 \mathrm{~cm}^{-3}$ represents the trough, and the range $0.1-$ $1 \mathrm{~cm}^{-3}$ represents the plasma sheet. This is a rough classification since, for instance, in the plasma sheet the density may exceed $1 \mathrm{~cm}^{-3}$ during magnetically active periods. This exercise should preferably be taken as an examination of what densities are typically encountered at geostationary orbit at different MLT sectors.

Based on the observed distributions, the plasma sheet is encountered quite often except for the 11-15 MLT sector. The probability of observing the plasma sheet correlates strongly with $K_{p}$ in the pre-midnight sector, whereas in the post-midnight sector the probability is approximately $25 \%$, with no significant $K_{p}$ dependence.

The top two panels in Fig. 8 can be used to predict how often the plasmapause extends beyond geosynchronous distance. The normal plasmaspheric densities $\left(10-100 \mathrm{~cm}^{-3}\right)$ are encountered frequently at all MLT, between a $30 \%$ probability near midnight and a $60 \%$ probability in the noondusk sector for low $K_{p}$; the highest probability of $70 \%$ appears near 16 MLT. Notice that the probability is roughly constant with MLT for a low $K_{p}$, whereas for a higher $K_{p}$ the probability has a strong MLT dependence. This is in accordance with the data shown in Fig. 4 which indicates that the plasmasphere moves earthward and becomes more asymmetric with increasing $K_{p}$. Notice that the dense plasmasphere $\left(>100 \mathrm{~cm}^{-3}\right)$ is observed at the geostationary distance only $10-15 \%$ of the time, except around 24 MLT, where the probability is almost zero. Such densities can be detected during intervals where the flux tubes have reached their saturation levels (Lawrence et al., 1999).

The trough is very commonly observed at 00-11 MLT with a clear $K_{p}$ dependence. On the other hand, in the dusk sector, the probability of encountering the trough shows no $K_{p}$ dependence, yet it increases towards midnight. In general, the probability of observing the trough appears to evolve inversely to that of the normal plasmapause.

\section{Summary}

Spacecraft potential measurements gathered with the Polar electric field instrument have been used to study the distribution of the electron density and its variations in the magnetosphere between $2-9 R_{E}$ geocentric distances. This technique provides a measure of the thermal plasma density to values even below $0.1 \mathrm{~cm}^{-3}$ with high time resolution (e.g. less than $0.1 \mathrm{~s})$.

Using this approach, global average densities were provided where signatures of the polar cap, cusp, and plasmapause are readily apparent, as well as their dependence on magnetic activity and season. Detailed studies of each area promise to elucidate many of the processes affecting the plasma distributions in these regions, particularly when analyzed in conjunction with electric and magnetic fields and particle data.

The major findings of the present study are:

(1) The density in the polar cap behaves systematically with season. At low altitudes $\left(\sim 1 R_{E}\right)$, the average density is approximately $100 \mathrm{~cm}^{-3}$ in summer and $1 \mathrm{~cm}^{-3}$ in winter. At high altitudes $\left(4-8 R_{E}\right)$, the density varies similarly with season between $0.03-0.3 \mathrm{~cm}^{-3}$. In fact, the polar cap density depends particularly upon whether or not the Sun is illuminating the footpoint region in the ionosphere.

(2) The geomagnetic effects are very important for the polar cap density: during high $K_{p}\left(\geq 4^{-}\right)$, the average density increases one or two orders of magnitude compared to the quiet-time level $\left(K_{p}<1\right)$. For individual events, corresponding to high magnetic activity, density enhancements can be much larger. The average wintertime density for high $K_{p}$ can easily exceed the average summertime density for low $K_{p}$. 
(3) The polar cap density declines quite steeply with altitude: in winter the density decreases by a factor of 10 20 from 2 to $4-8 R_{E}$ altitudes. In summer, the decline is much stronger, of the order of 200-1000, over the same altitude range. A detailed analysis for quiet intervals in winter suggests that the density varies with altitude as $r^{-4.4}$, implying that the ionospheric plasma is accelerated with distance as $r^{1.4}$.

(4) The cusp plasma density and location can significantly change from orbit to orbit. The average cusp is $1-2^{\circ}$ wide and centered at $80-82^{\circ}$ for quiet intervals, and moves equatorward for increasing $K_{p}$. For high $K_{p}$, the cusp disappears in the average pictures to be likely due to a large spread of the cusp location under disturbed conditions.

(5) Analysis of the densities near $\mathrm{L}=6.6$ shows that the average density obtained here is in good accordance with daily variations reported by Higel and Wu (1984). In addition, we found that the average density decreases with increasing $K_{p}$ at all MLT sectors, except 14-17 MLT. The large density values in this sector are possibly maintained by the rotation of the bulge toward the Sun or detached plasma elements eroded from the bulge region.

(6) The electron density distribution at $\mathrm{L}=6.6$ indicates that the dense plasmasphere $\left(>100 \mathrm{~cm}^{-3}\right)$ is detected $0-15 \%$ of the time, depending on MLT and $K_{p}$. The normal plasmasphere $\left(10-100 \mathrm{~cm}^{-3}\right)$ is detected quite frequently, with a strong dependence on MLT and $K_{p}$. As an example, for $K_{p}=0-1$, the probability is 30-60\%, depending on MLT, while the probability is only 10$40 \%$ for $K_{p} \geq 3^{-}$. The trough $\left(1-10 \mathrm{~cm}^{-3}\right)$ is encountered as frequently as the normal plasmasphere, but the probability evolves with MLT and $K_{p}$ inversely to that of the normal plasmapause. The highest probabilities for detecting the trough are 65-80\% at 00-11 MLT for high $K_{p}$. Low densities $\left(0.1-1 \mathrm{~cm}^{-3}\right)$, related to plasma sheet encounters, are detected most likely in the 1824 MLT sector, with probabilities increasing with $K_{p}$. For high $K_{p}$, the plasma sheet is encountered approximately $50 \%$ of the time. At 00-06 MLT, the probability is $20 \%$, with no apparent $K_{p}$ dependence.

Acknowledgements. This work is supported under NASA grant NAG5-3182.

Topical Editor G. Chanteur thanks G. Marklund and V. Pierrara for their help in evaluating this paper.

\section{References}

Carpenter, D. L., Giles, B. G., Chappell, C. R., Decreau, P. M. E., Anderson, R. R., Persoon, A. M., Smith, A. J., Corcuff, Y., and Canu, P.: Plasmasphere dynamics in the duskside bulge sector: a new look at an old topic, J. Geophys. Res., 98, 19243-19271, 1993.

Chappell, C. R.: Detached plasma regions in the magnetosphere, J. Geophys. Res., 79, 1861-1870, 1974.
Escoubet, C. P., Pedersen, A., Schmidt, R., and Lindqvist, P. A.: Density in the magnetosphere inferred from ISEE-1 spacecraft potential, J. Geophys. Res., 102, 17 595-17 609, 1997.

Gallagher, D. L., Craven, P. D., Comfort, R. H., and Moore, T. E.: On the azimuthal variation of core plasma in the equatorial magnetosphere, J. Geophys. Res., 100, 23 597-23 605, 1995.

Harvey, P., Mozer, F. S., Pankow, D., Wygant, J., Maynard, N. C., Singer, H., Sullivan, W., Anderson, P. B., Pfaff, R., Aggson, T., Pedersen, A., Falthammar, C.-G., and Tanskanen, P.: The electric field instrument on the Polar satellite, Space Sci. Rev., 71, 583596, 1995.

Higel, B. and Wu Lei: Electron density and plasmapause characteristics at $6.6 \mathrm{Re}$ : a statistical study of the GEOS 2 relaxation sounder data, J. Geophys. Res., 89, 1583-1601, 1984.

Johnson, M. T., Wygant, J. R., Cattell, C., Mozer, F. S., Temerin, M., and Scudder, J.: Observations of the seasonal dependence of the thermal plasma density in the Southern Hemisphere auroral zone and polar cap at 1 Re, J. Geophys. Res., 106, 19 023-19033, 2001.

Kurita, K. and Hasegawa, M.: Evaluation of the effectiveness of theoretical model calculation in determining the plasmapause structure, J. Geophys., 57, 103, 1985.

Laakso, H. and Grard, R.: The electron density distribution in the polar cap: its variability with seasons, and its response to magnetic activity, in: Space Weather Study Using Multipoint Techniques, (Ed) Lyu, L.-H., Proceedings of COSPAR Colloquium 12, Pergamon, 193-202, 2002.

Laakso, H. and Jarva, M.: Position and motion of the plasmapause, J. Atmos. Terr. Sol. Phys., 63, 1171-1178, 2001.

Laakso, H. and Pedersen, A.: Ambient electron density derived from differential potential measurements, in: Measurement Techniques in Space Plasmas, (Eds) Borovsky, J., Pfaff, R., and Young, D., AGU Monograph 102, AGU, Washington, DC, 4954, 1998.

Laakso, H., Opgenoorth, H., Wygant, J., Escoubet, P., Clemmons, J., Johnson, M., Maynard, N., Mozer, F., Pfaff, R., and Scudder, J.: Electron Density Distribution in the Magnetosphere, in: Correlated Phenomena at the Sun, in the Heliosphere and in Geospace, ESA SP-15, ESA, Noordwijk, The Netherlands, 5358, 1997.

Laakso, H., Pfaff, R., and Janhunen, P.: Polar observations of electron density distribution in the Earths magnetosphere. 2. Density profiles, Ann. Geophysicae, this issue, 2002.

Lawrence, D. J., Thomsen, M. F., Borovsky, J. E., and McComas, D. J.: Measurements of early and late time plasmasphere refilling as observed from geosynchronous orbit, J. Geophys. Res., 104, 14 691-14 704, 1999.

Lemaire, J. F. and K. I. Gringauz, The Earth's Plasmasphere, Cambridge Univ. Press, Cambridge, 1998.

Lyons L. R. and Williams, D. J.: Quantitative Aspects of Magnetospheric Physics, D. Reidel, Dordrecht, 1984.

Marklund, G. T., Blomberg, L. G., Falthamma, C.-G., Erlandson, R. E., and Potemra, T. A.: Signatures of the high-altitude polar cusp and dayside auroral regions as seen by the Viking electric field experiment, J. Geophys. Res., 95, 5767-5780, 1990.

Moore, T. E. and Delcourt, D. C.: The geopause, Rev. Geophys., 33, 175-209, 1995.

Moore, T. E., Chappell, C. R., Chandler, M. O., Fields, S. A., Pollock, C. J., Reasoner, D. L., Young, D. T., Burch, J. L., Eaker, N., Waite, J. H., McComas, D. J., Norholt, J. E.,Thomsen, M. F., Berthelier, J. J., and Robson, R.: The thermal ion dynamics experiment and plasma source instrument, Space Sci. Rev., 71, 409, 
1995.

Mozer, F. S., Torbert, R. B., Fahleson, U. V., Fálthammar, C.-G., Gonfalone, A., and Pedersen, A.: Electric field measurements in the solar wind, bow shock, magnetosheath, magnetopause, and magnetosphere, Space Sci. Rev., 22, 791-804, 1978.

Ober, D. M., Horwitz, J. L., Thomsen, M. F., Elphic, R. C., McComas, D. J., Belian, R. D., and Moldwin, M. B.: Premidnight plasmaspheric "plumes", J. Geophys. Res., 102, 11 325-11 334, 1997.

Palmroth, M., Laakso, H., and Pulkkinen, T.: Location of highaltitude cusp during steady solar wind conditions, J. Geophys. Res., 106, 21 109-21 122, 2001.

Pedersen, A.: Solar wind and magnetosphere plasma diagnostics by spacecraft electrostatic potential measurements, Ann. Geophysicae, 13, 118-129, 1995.

Pedersen, A., Grard, R., Knott, K., Jones, D., Gonfalone, A., and Fahleson, U.: Measurements of quasistatic electric fields between 3 and 7 Earth radii on GEOS-1, Space Sci. Rev., 22, 333346, 1978.
Pedersen, A., Cattell, C. A., Fa̋lthammar, C.-G., Formisano, V., Lindqvist, P.-A., Mozer, F., and Torbert, R.: Quasistatic electric field measurements with spherical double probes on the GEOS and ISEE satellites, Space Sci. Rev., 37, 269-312, 1984.

Persoon, A. M., Gurnett, D. A., and Shawhan, S. D.: Polar cap electron densities from DE 1 plasma wave observations, J. Geophys. Res., 88, 10 123-10 136, 1983.

Rosenbauer, H., Grûnwaldt, H., Montgomery, M. D., Paschmann, G., and Sckopke, N.: Heos 2 observations in the distant polar magnetosphere: the plasma mantle, J. Geophys. Res., 80, 27232737, 1975.

Scudder, J. D., Cao, X., and Mozer, F. S.: The photoemission current - spacecraft potential relation: Key to routine, quantitative low-energy plasma measurements, J. Geophys. Res., 105, $21281-21294,2000$.

Tsyganenko, N. A. and Stern, D. P.: Modeling the global magnetic field of the large-scale Birkeland current systems J. Geophys. Res., 101, 27 187-27 198, 1996. 